\title{
The Research on Level Set Segmentation Method Based on Kernel Principal Component Analysis Prior Shape
}

\author{
Ji Zhao*, Huibin Wang and Xuefeng Li \\ School of Software Engineering \\ University of Science and Technology Liaoning \\ Anshan 114051, China \\ zhaoji_1974@126.com
}

\begin{abstract}
It is often a difficult task to accurately segment image with weak borders or complex background, in view of seriousness of the task, this paper proposes a new face segmentation method based on level set prior shape. In this paper, the proposed method maps the samples into feature space, uses the kernel principal component analysis algorithm to reduce the dimension of the samples, extracts the principal component as the prior shape to guide the image segmentation, and does morphological processing on the mean shape which is used as the initial contour to effectively solve the determined problem of the initial contour of the evolution curve. The priori shape energy term is introduced to the level set and the local binary fitting model, which can effectively improve the speed and accuracy of image segmentation. Several experiments are provided to illustrate the efficiency of the proposed methods.
\end{abstract}

Keywords: Face segmentation, Prior shape, Initial contour, local binary fitting, Level set

\section{Introduction}

In the last decade, image segmentation gained a lot of interest, it has always been an important field of computer vision but not yet fully resolved. Image segmentation technique has been applied to many areas of real life, such as video tracking, traffic management, identification, medical image segmentation, and so on. Active contour models[1] have been one of the most successful methods in the field of image segmentation.

There are two broad categories in the active contour models, such as the edge-based active contour models[1] and the region-based active contour models[2]. The edge-based active contour models utilizes the gradient information of the image to make the evolving contour curve stop at the target boundary. For example, Caselles et al[3] put forward the classic geodesic active contour model. And the region-based active contour model utilizes global information. Mumford D and Shah J[4] proposed the Mumford-Shah model which is based on the region. Chan and Vese improved the Mumford-Shah model and proposed the C-V Model[6]. In the literature[5], Vese and Chan used the multiphase level set framework to improve the $\mathrm{C}-\mathrm{V}$ mode, and proposed the Piecewise Constant Models. Li C, Kao C Y and Gore J C[7] introduced the local information of an image into the region segmentation model, proposed the local binary fitting model (LBF Model).

Some improved image segmentation algorithms has obtained the good effects in various areas of image segmentation, but can't achieve satisfied results when using the segmentation model individually for some more complex images, for example the target is obscured either damaged or the gray scale of the target and the background are very

* Corresponding Author 
close. So it becomes an important research content of the level set segmentation to integrate the prior knowledge into the segmentation model. P. H. Lim et al[8] proposed a method combined the prior shape and Willmore Flow to drive the level set evolve which successfully applied it to spinal CT image segmentation. S. Chen et al[9] proposed a novel variational level set segmentation framework, which not only combining the information of the prior intensity and shape but also dividing the total energy functional into shape functional part and image energy functional part. V. A. Prisacariu et al[10] used the Elliptical Fourier Descriptors to express shape and Gaussian Process Latent Variable Models to learn their low dimensional potential space. O. Gloger [11] applied Bayesian concept to probability graph generating, following this he proposed an extended level set method based on prior shape. F. Khalifa[12] proposed a novel velocity function to guide the active contour curve evolution which is based on level set. S. Y. Yeo[13] proposed a novel level set segmentation method which uses statistical prior shape of variational method. Shah[14] proposed an effective variational level set framework which uses statistical priori shape to restore shape descriptors from the occluded target. J. Y. Dong[15] proposed priori shape segmentation model based on principal component analysis and maximum a posteriori. X. J. Zheng[16] proposed a segmentation model based on shape prior and Mumford-Shah model which utilizes principal component analysis algorithm to obtain the main information of the interesting object's shape. L. P. Yang[17] proposed a novel image segmentation method based on shape prior which uses variational level set method, combines regional characteristics and boundary contour features and chooses the best prior shape via similarity matching.

Based on the above discussion, the prior shape is introduced into the model to improve the segmentation results. The principal component analysis (PCA) algorithm is mainly introduced to extract the principal components in the samples. Owing to the PCA algorithm is expressed by a linear shape prior term which can't represent the shape accurately, the final segmentation result is not ideal. In order to overcome the limitation of level set framework based on PCA algorithm, we use Kernel Principal Component Analysis method to reduce the dimension of training samples to solve the malpractice of PCA method. In the interest of improving the initial contour of level set segmentation, we solve the mean of sample signed distance function by doing the expansion process on the sample mean which is regarded as the initial contour of level set segmentation. We also adopt the improved LBF model, furthermore introducing the priori shape item which can improve the efficiency and accuracy of image segmentation.

\section{Theoretical Knowledge}

\subsection{Improve LBF Model}

LBF (Local Binary Fitting) model is an energy fitting model of regional extensibility. The range of the fitting grey value area is determined by the size of parameters of the introduced non-negative kernel function. For any of $x \in \Omega$ ( $\Omega$ is the image area), the energy functional of LBF is as following:

$$
E_{x}=\lambda_{1} \int_{\text {in }(C)} K(x-y)\left|I(y)-f_{1}(x)\right|^{2} d y+\lambda_{2} \int_{\text {out }(C)} K(x-y)\left|I(y)-f_{2}(x)\right|^{2} d y
$$

where $\lambda_{1}, \lambda_{2}$ are positive constants, $K(x)$ is the weighting function. Meeting the local nature: non-negative and monotone decreasing, $\lim _{|u| \rightarrow 0} K(u)=0, f_{1}(x)$ and $f_{2}(x)$ are the fitting values near $\mathrm{x}$ in the image. There are many weighting functions which meet (1), we define weighting function $\mathrm{K}(\mathrm{u})$ as the Gaussian Kernel Function which is defined by: 


$$
K_{\sigma}(u)=\frac{1}{(\sqrt{2 \pi} \sigma)^{n}} e^{-|u|^{2} / 2 \sigma^{2}}
$$

where $\sigma>0$.

However, $E_{x}$ is defined about each of center points $x \in \Omega$ locally in formula (1). We must minimize the energy $E_{x}$ on all of center points $x$ in the whole image area $\Omega$ in order to determine the target boundary. The total energy functional is defined by:

$E^{L B F}=\int_{\Omega} E_{x}\left(C, f_{1}(x), f_{2}(x)\right) d x$

In order to avoid the periodic re-initialization of level set, Li. C[1] introduced the method without re-initialization into the LBF model. besides, we introduce Heaviside Function and Dirac Function into variational level set methods, so formula (1) is rewritten to the fitting energy functional of zero level set function, which is defined by:

$$
\begin{gathered}
F\left(\phi, f_{1}, f_{2}\right)=\lambda_{1} \int\left[\int K_{\sigma}(x-y)\left|I(y)-f_{1}(x)\right|^{2} H(\phi(y)) d y\right] d x \\
+\lambda_{2} \int\left[\int K_{\sigma}(x-y)\left|I(y)-f_{2}(x)\right|(1-H(\phi(y))) d y\right] d x \\
+\beta \int_{\Omega} \frac{1}{2}(|\nabla \phi(x)|-1)^{2} d x+v \int_{\Omega} \delta(\phi(x))|\nabla \phi(x)| d x
\end{gathered}
$$

where $\phi_{\text {denotes the zero level set function. }}$

In the experiments, Heaviside Function is replaced by smoothing function approximatively in (4), which is defined by:

$H_{\varepsilon}(x)=\frac{1}{2}\left[1+\frac{2}{\pi} \arctan \left(\frac{x}{\varepsilon}\right)\right]$

The derivative of $H_{\varepsilon}$ is defined as following, which is called as $\delta_{\varepsilon}(x)$.

$\delta_{\varepsilon}(x)=H_{\varepsilon}^{\prime}(x)=\frac{1}{\pi} \frac{\varepsilon}{\varepsilon^{2}+x^{2}}$

For a fixed level set function: $\phi$, we minimize (4) and gain:

$f_{1}(x)=\frac{K_{\sigma}(x) *\left[H_{\varepsilon}(\varphi(x)) I(x)\right]}{K_{\sigma}(x) * H_{\varepsilon}(\varphi(x))}$

$f_{2}(x)=\frac{K_{\sigma}(x) *\left[\left(1-H_{\varepsilon}(\varphi(x))\right) I(x)\right]}{K_{\sigma}(x) *\left[1-H_{\varepsilon}(\varphi(x))\right]}$

$f_{1}(x)$ and $f_{2}(x)$ being kept unchanged, minimize the energy functional $F\left(\phi, f_{1}, f_{2}\right)$ and get the following evolution equation by using the gradient descent method.

$$
\begin{aligned}
\frac{\partial \varphi}{\partial t}= & -\delta_{\varepsilon}(\varphi)\left(\lambda_{1} e_{1}-\lambda_{2} e_{2}\right)+v \delta_{\varepsilon}(\varphi) \operatorname{div}\left(\frac{\nabla \varphi}{|\nabla \varphi|}\right) \\
& +\beta\left(\nabla^{2} \varphi-\operatorname{div}\left(\frac{\nabla \varphi}{|\nabla \varphi|}\right)\right)
\end{aligned}
$$

The computational formula of $e_{1}$ and $e_{2}$ are as follows: 


$$
\begin{aligned}
& e_{1}(x)=\int_{\Omega} K_{\sigma}(y-x)\left|I(x)-f_{1}(y)\right|^{2} d y \\
& e_{2}(x)=\int_{\Omega} K_{\sigma}(y-x)\left|I(x)-f_{2}(y)\right|^{2} d y
\end{aligned}
$$

\subsection{Kernel Principal Component Analysis}

Mika[20] proposed kernel principal component analysis for the first time which has a strong advantage in extracting nonlinear structure of the data set. The basic idea of kernel principal component analysis is mapping data sets from input space I to feature space $\mathrm{F}$ through a nonlinear mapping function: $\varphi$. Then, we can perform PCA algorithm in the feature space and find the principal components of a data set corresponding to the largest changed part of the map data set. The solved $l$ principal components will use $l$ directions to explain the changes of variances in the data set as much as possible.

The nonlinear mapping function $\varphi: I \rightarrow F$ typically does not need to be known through the use of Mercer kernel whose function written as $k(.,$.$) . For all data points x_{i}$, the kernel matrix is symmetric positive definite. According to Mercer's Theory [18], solving $k(.,$.$) is equivalent to compute the inner scalar product$ in $F: k\left(x_{a}, x_{b}\right)=\left(\varphi\left(x_{a}, x_{b}\right)\right)$, with $\left(x_{a}, x_{b}\right) \in I \times I$. A distance in the feature space is defined by

$$
\begin{aligned}
d_{F}^{2}\left(\phi\left(x_{a}\right), \phi\left(x_{b}\right)\right)=\left\|\phi\left(x_{a}\right)-\phi\left(x_{b}\right)\right\|^{2} \\
=k\left(x_{a}, x_{a}\right)-2 k\left(x_{a}, x_{b}\right)+k\left(x_{b}, x_{b}\right)
\end{aligned}
$$

Now we briefly describe the KPCA method [20]. Let $X=\left\{x_{1}, x_{2}, \ldots, x_{N}\right\}$ be a set of

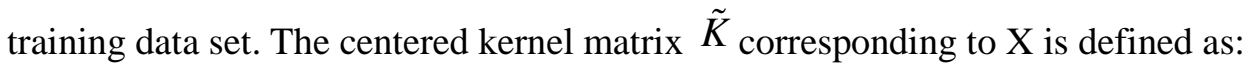

$$
\begin{aligned}
\tilde{K} & =\left(\left(\phi\left(x_{i}\right)-\bar{\phi}\right) \cdot\left(\phi\left(x_{j}\right)-\bar{\phi}\right)\right) \\
& =\left(\tilde{\phi}\left(x_{i}\right) \cdot \tilde{\phi}\left(x_{j}\right)\right)=\tilde{k}\left(x_{i}, x_{j}\right), \quad i, j=1,2, \ldots, N
\end{aligned}
$$

With $\bar{\varphi}=\frac{1}{N} \sum_{i=1}^{N} \varphi\left(x_{i}\right), \tilde{\varphi}\left(x_{i}\right)=\varphi\left(x_{i}\right)-\bar{\varphi}$ being the centered map corresponding to $x_{i}$ and $\tilde{k}(\cdot, \cdot)$. At last, the kernel matrix $\tilde{K}$ is revised as follows

$$
\begin{aligned}
K L_{i j} & =\tilde{k}_{i j}-\frac{1}{N}\left(\sum_{\mu=1}^{N} \tilde{k}_{i \mu}+\sum_{\mu=1}^{N} \tilde{k}_{\mu j}\right) \\
& +\frac{1}{N^{2}} \sum_{\mu, v=1}^{N} \tilde{k}_{\mu v}
\end{aligned}
$$

with $\tilde{k}_{i j}=\tilde{k}\left(x_{i}, x_{j}\right), i, j=1,2, \ldots, N$. The revised matrix is written as $K L$. Since $K L$ is symmetric, it can be decomposed as:

$$
K L=U S U^{T}
$$

where $S=\operatorname{diag}\left(\lambda_{1}, \lambda_{2}, \ldots, \lambda_{N}\right)$ is a diagonal matrix containing the eigenvalues of $K L . U=\left[\vec{u}_{1}, \vec{u}_{2}, \ldots, \vec{u}_{N}\right]$ is the orthogonal matrix, each column vectors $\vec{u}_{i}=\left[u_{i 1}, \ldots, u_{i N}\right]$ are the eigenvectors corresponding to the eigenvalues $\lambda_{i}$. In addition, the centered kernel 
matrix can be expressed as $K L=H K H$, where $H=E-\frac{1}{N} l l^{T} . l=[1,1, \ldots, 1]^{T}$ is an $N \times 1$ vector, $E$ is an identity matrix.

Let $C$ denote the covariance matrix of all elements of the training set which is mapped by $\tilde{\varphi}$. Due to it is based on kernel principal component analysis methodology, the covariance matrices $C$ does not need to be solved directly. Only $K L$ needs to be solved to extract the characteristics of the training sample because the eigenvectors of $\mathrm{C}$ are the simple function of the eigenvectors of $K L$. The KPCA space is the subspace of $F$ obtained from learning the training data.

Let $x$ be the arbitrary element in the input space $I$. The projection of $x$ on the KPCA space can be denoted by $p^{l} \varphi(x)$. The detailed description of $p^{l} \varphi(x)$ can be referred to[20]. The squared distance $d_{F}^{2}$ between the mapping of $x$ mapped by $\varphi$ and its projection $p^{l} \varphi(x)$ on the KPCA space is given by

$$
\begin{aligned}
& d_{F}^{2}\left[\phi(x), P^{l} \phi(x)\right]=\left\|\phi(x)-P^{l} \phi(x)\right\|^{2} \\
& \quad=k(x, x)-2\left(\phi(x) \cdot P^{l} \phi(x)\right)+\left(P^{l} \phi(x) \cdot P^{l} \phi(x)\right)
\end{aligned}
$$

$d_{F}^{2}$ measures the discrepancy between a (mapped) element of $I$ and the elements of the learned space and will be minimized to introduce shape knowledge in the contour evolution process, and the specific narrative will be described in Section 3. In accordance with the relevant matrix manipulations, this squared distance can be expressed only in terms of kernels as

$$
\begin{aligned}
d_{F}^{2}\left[\varphi(x), p^{l} \varphi(x)\right] & =k(x, x)+\frac{1}{N^{2}} l^{T} K l-\frac{2}{N} l^{T} k_{x} \\
& +\tilde{k}_{x}^{T} M K L M \tilde{k}_{x}-2 \tilde{k}_{x}^{T} M \tilde{k}_{x}
\end{aligned}
$$

where $\quad k_{x}=\left[k\left(x, x_{1}\right), k\left(x, x_{2}\right), \ldots, k\left(x, x_{N}\right)\right]^{T} \quad, \quad \tilde{k}_{x}=H\left(k_{x}-\frac{1}{N} K l\right)$, $M=\sum_{i=1}^{l} \frac{1}{\lambda_{i}} u_{i} u_{i}^{T}$, and $l=[1, \ldots, 1]^{T}$ is a $N \times 1$ vector. $K$ and $K L$ respectively denote the kernel matrix and the revised matrix.

It is the basis of the kernel principal component analysis algorithm which choose a nonlinear kernel function to the KPCA method. The commonly used kernel function mainly includes Polynomial Kernel Function, Gaussian Radial Kernel Function, Multilayer Perceptron Kernel Function and Dynamic Kernel Function, which are respectively defined:

Polynomial Kernel Function

$$
K(x, y)=[(x \cdot y)+1]^{p}
$$

Gaussian Radial Kernel Function

$$
K(x, y)=\exp \left(-\frac{\|x-y\|^{2}}{2 \sigma^{2}}\right)
$$

Multi-layer Perceptron Kernel Function

$$
K(x, y)=\tanh [v(x \cdot y)+c]
$$

Since Gauss Radial Kernel Function can preferably extract nonlinear features[19] from the data set and has become a more popular kernel function in the field of machine 
learning, we choose Gaussian Radial Kernel as the nonlinear kernel function. In this paper, $\mathrm{SDF}$ is adopted to denote the introduced shape. The revised kernel function is defined by

$k_{\phi_{\sigma}}\left(\varphi_{i}, \varphi_{j}\right)=e^{\frac{-\left\|\varphi_{i}-\varphi_{j}\right\|^{2}}{2 \sigma^{2}}}$

where $\sigma^{2}$ is variance parameter, $\left\|\phi_{i}-\phi_{j}\right\|^{2}$ is the L2-distance between $\phi_{i}$ and $\phi_{j}$. Subscript denotes the nonlinear mapping in according with Gaussian Radial Kernel Function which also depends on the selection of parameters.

\section{The Novel Model Improved in this Paper}

\subsection{Priori Shape Item}

In order to introduce the prior shape items into the improved LBF model, we adopt the thought of [21]. We define the shape energy functional in KPCA mapping space as follows

$$
E_{\text {shape }}^{F}(\varphi):=d_{F}^{2}\left[\phi(\varphi), P^{l} \phi(\varphi)\right]
$$

$\phi$ denotes the SDF of the training sample, and the specific description of $p^{l} \varphi(x)$ is referred to [22]. The superscript $\mathrm{F}$ of $E_{\text {shape }}^{F}$ denotes that the knowledge of shape is described as the distance in the feature space.

We use variational method which introduce Gaussian Radial Kernel Function. We define the gradient of $E_{\text {shape }}^{F}$

$\nabla_{\varphi} E_{\text {shape }}^{F}=-\frac{\sum_{i=1}^{N} g_{i}(\varphi) \cdot k\left(H(\varphi), H\left(\varphi_{i}\right)\right) \cdot \delta(\varphi) \cdot\left[H(\varphi)-H\left(\varphi_{i}\right)\right]}{\sigma^{2}}$

where

$$
\begin{aligned}
{\left[g_{1}(\varphi), g_{2}(\varphi), \ldots, g_{N}(\varphi)\right] } & =-\frac{2}{N} l^{T}+2 \tilde{k}_{\varphi}^{T} M K L M H \\
& -4 \tilde{k}_{\varphi}^{T} M H
\end{aligned}
$$

The specific solved method of $\tilde{k}_{\phi}, M, K L$ has been described in the above chapter in detail.

Because $E_{\text {shape }}^{F}$ is based on feature space, it is necessary to firstly deform $E_{\text {shape }}^{F}$ to convert the energy item of the feature space to the energy item of the input space for better integration with image energy.

Now we define the relationship between the distance in the feature space and the distance in the input space as follows

$$
\begin{aligned}
d_{F}^{2}\left(\varphi_{a}, \varphi_{b}\right) & =2-2 k\left(H\left(\varphi_{a}\right), H\left(\varphi_{b}\right)\right) \\
& =2-2 e^{\frac{-\left\|H\left(\varphi_{a}\right)-H\left(\varphi_{a}\right)\right\|^{2}}{2 \sigma^{2}}} \\
& =2-2 e^{-\frac{d_{I}^{2}\left(H\left(\varphi_{a}\right), H\left(\varphi_{b}\right)\right)}{2 \sigma^{2}}}
\end{aligned}
$$


where $d_{I}^{2}$ denotes a squared distance in the input space, $k(\cdot, \cdot)$ is Gaussian Radial Kernel Function and $H(\phi)$ is Heaviside Function. Because of the invertibility of the kernel, we can obtain as follows

$d_{I}^{2}\left(H\left(\varphi_{a}\right), H\left(\varphi_{b}\right)\right)=-2 \sigma^{2} \log \left(\frac{2-d_{F}^{2}\left(\varphi_{a}, \varphi_{b}\right)}{2}\right)$

Now we define a new shape energy form $E_{\text {shape }}^{F}$ :

$E_{\text {shape }}=-2 \sigma^{2} \log \left(\frac{2-E_{\text {shape }}^{F}}{2}\right)$

By applying the chain rule, the gradient of $E_{\text {shape }}$ is defined by:

$\nabla_{\phi} E_{\text {shape }}=\frac{2 \sigma^{2}}{2-E_{\text {shape }}^{F}} \nabla_{\phi} E_{\text {shape }}^{F}$

where $E_{\text {shape }}$ is computed via (25), $E_{\text {shape }}^{F}$ is computed via (20).

\subsection{Combining Image Information and Shape Priori}

In this section, we will combine shape prior and image information which adopts the improved LBF model. The total energy is defined by

$E_{\text {total }}=(1-\alpha) E_{\text {image }}+\alpha E_{\text {shape }}$

where $\alpha$ is the weighting coefficient.

By introducing the regularized Heaviside function and Dirac function, (21) can be approximately defined by

$E_{\text {total }}=(1-\alpha)\left(E_{\varepsilon}^{L B F}+\beta D(\varphi)+v L_{\varepsilon}(\varphi)\right)+\alpha E_{\varepsilon}^{\text {shape }}$

By minimizing the total energy, we can obtain the gradient descent flow of (22) which is shown as follows

$$
\begin{aligned}
& \frac{\partial \phi}{\partial t}=(1-\alpha)\left(-\delta_{\varepsilon}(\phi)\left(\lambda_{1} e_{1}-\lambda_{2} e_{2}\right)+v \delta_{\varepsilon}(\phi) \operatorname{div}\left(\frac{\nabla \phi}{|\nabla \phi|}\right)\right. \\
& \left.+\beta\left(\nabla^{2} \phi-\operatorname{div}\left(\frac{\nabla \phi}{|\nabla \phi|}\right)\right)\right)+ \\
& \alpha\left(\frac{2 \sigma^{2}}{2-E_{\text {shape }}^{F}}\left(-\frac{\sum_{i=1}^{N} g_{i}(\phi) k\left(H_{\varepsilon}(\phi), H_{\varepsilon}\left(\phi_{i}\right)\right) \cdot \delta_{\varepsilon}(\phi) \cdot\left[H_{\varepsilon}(\phi)-H_{\varepsilon}\left(\phi_{i}\right)\right]}{\sigma^{2}}\right)\right)
\end{aligned}
$$

\section{Algorithm Implementation}

\subsection{Obtaining the Sample Sets}

This experiment chooses the representative 18 face images as the samples, and does binarization processing on each sample which are shown as follows 


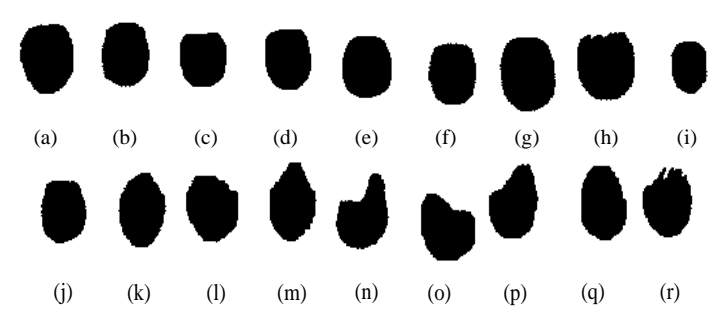

Figure 1. Original Samples

The training sample set is composed of 18 binary images in Figure 1, written as $\chi=\left\{\chi_{1}, \chi_{2}, \ldots, \chi_{18}\right\}$. The pixel values of the target is 0 , and the pixel values of the background is 1 in each sample image.

The method used in this paper to construct the priori shape is as follows: we rectify the binary images of the training samples, then use the formula of SDF[22] to solve the SDFs of the binary images after registration. The specific steps of the registration includes: translation, rotation and scaling, while translation formula is defined by

$$
x^{k}=\frac{\sum_{i=1}^{m} \sum_{j=1}^{n} x_{i} \chi_{k}\left(x_{i}, y_{j}\right)}{\sum_{i=1}^{m} \sum_{j=1}^{n} \chi_{k}\left(x_{i}, y_{j}\right)}, \quad y^{k}=\frac{\sum_{i=1}^{m} \sum_{j=1}^{n} x_{i} \chi_{k}\left(x_{i}, y_{j}\right)}{\sum_{i=1}^{m} \sum_{j=1}^{n} \chi_{k}\left(x_{i}, y_{j}\right)}
$$

where $k=1,2, \ldots, 18, m$ and $n$ respectively denote the number of rows and columns in a binary image, $\left(x_{i}, y_{i}\right)$ represents the coordinate which corresponds to the pixels in a binary image. $\chi_{k}$ denotes the $k t h$ binary image. After calculating barycentric coordinate of each sample binary image, we can solve the relevant translation volume which responds to each referred image. The scaling formula is defined by

$$
s^{k}=\frac{1}{L^{k}} \sum_{i=1}^{L^{k}} d\left(\left(x_{i}^{k}, y_{i}^{k}\right),\left(x^{k}, y^{k}\right)\right)
$$

where $d\left(\left(x_{i}^{k}, y_{i}^{k}\right),\left(x^{k}, y^{k}\right)\right)$ denotes the Euclidean distance between the coordinate $\left(x_{i}^{k}, y_{i}^{k}\right)$ on the edge of the target contour and the barycentric coordinate $\left(x^{k}, y^{k}\right)$. So the zoom scale of the kth sample binary image can be defined by $s^{k} / s^{l}$, where $s^{l}$ is the average distance between point on the contour edge of the chosen referred binary image and the barycentric coordinate of the image.

The final sample training set after translation, scaling and rotation is shown in Figure 2.

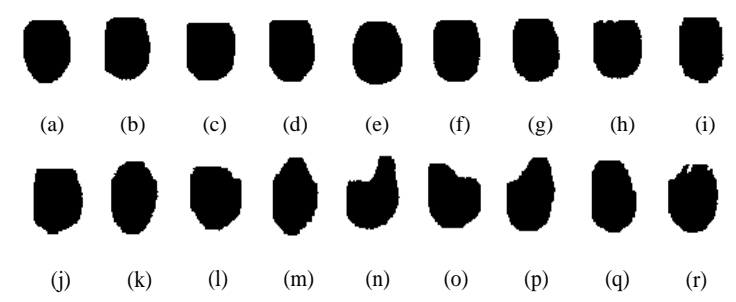

Figure 2. Final Training Samples 


\subsection{Using KPCA Method to Process Sample Set}

In order to reduce a large number of redundant information in the training sample, we use the KPCA method to decrease the dimensions of the SDFs of the training sample in this section.

Firstly, it is necessary to use formula of SDF to calculate the SDFs which correspond to the training sample shapes shown in Figure 2, and the SDFs of each training sample are shown in Figure 3.

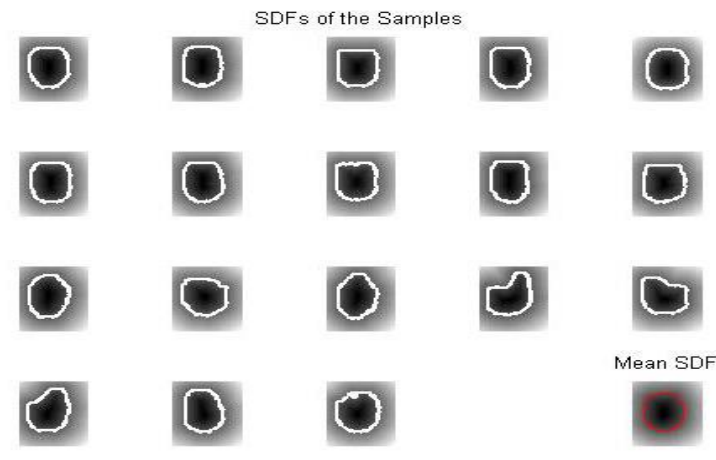

Figure 3. The SDFs of Training Samples

In Figure3, the closed white line denotes the contour line of each training samples shown in Figure 2, and the closed red line denotes the average contour of the SDFs of all the training samples. We do the expanding operations on the sample mean and translation on the average contour after expanding to make the contour be closer to target boundary. The contour is regarded as the initial contour before the formal segmentation to reduce the segmentation time and improve the segmentation accuracy.

So far, we obtain the SDFs of all the training samples, which are defined by $\phi_{1}, \phi_{2}, \ldots, \phi_{18}$. Since the SDF is only expressed by its corresponding to the zero level set, each symbol distance mapping must have a large number of redundant information. In order to reduce a lot of redundant information, Leventon[23] applied PCA method to the SDF of the contour, obtained the ideal segmentation results. But it used the linear form to restrict the new sample shapes in the process of using PCA method to deal the SDFs. There is a big limitation by using PCA method, because the actual shapes are not expressed by the linear relation. In order to solve the limitations caused by PCA method, kernel principal component analysis algorithm is adopted to decrease dimensions of the SDFs of the sample.

Before adopting the KPCA method to decrease dimensions of the SDFs of the samples, we firstly adjust the dimension of the SDFs of each sample. The specific way is that we adjust the data of each SDF into a $m n \times 1$ column vector, $m$ and $n$ respectively denote the number of rows and columns of each SDF. Then we form all SDFs to a new sample matrix $\mathrm{M}$, where $N=18$. Based on the basic principle of KPCA method described in Section 2, the processing steps of KPCA are as follows:

Choose the parameter $\sigma$ in Gaussian Radial Kernel Function, and calculate the kernel matrix $K$ by (10), whose inner product is replaced by Gaussian Radial Kernel Function. Then revise the kernel matrix by (11) and obtain $K L$.

Calculate the eigenvalues $\lambda_{1}, \lambda_{2}, \ldots, \lambda_{N}(N=m n)$ and their corresponding eigenvectors $v_{1}, v_{2}, \ldots, v_{N}(N=m n)$ of $K L$ by applying Jacobi iteration algorithm. 
Sort the eigenvalues in descending order (via Choice Sort) then obtain $\lambda_{1}^{\prime}>\lambda_{2}^{\prime}>\ldots>\lambda_{N}^{\prime}$, and adjust their corresponding eigenvectors then obtain $v_{1}^{\prime}, v_{2}^{\prime}, \ldots, v_{N}^{\prime}$.

Orthogonalize eigenvectors which is obtained in (2) via Schmidt Orthogonalization method then obtain $\alpha_{1}, \alpha_{2}, \ldots, \alpha_{N}$.

Calculate the cumulative contribution rate of the eigenvalues. According to a given ratio $p$, if $B_{t}>p$, extract t principal components: $\alpha_{1}, \alpha_{2}, \ldots, \alpha_{t}$.

Calculate the projection of the revised kernel matrix $K L$ on the extracted eigenvectors according to the extracted principal components.

In algorithm implementation process, the degree of fitting adopted in the experiment is $p=0.98$. After decreasing dimensions of the SDFs by KPCA method, $t$ meeting the requirements is equal to11. In this way, the original $18 \mathrm{SDFs}$ of the samples is replaced by the reconstructed 11 SDFs of the samples which are used as a new sample prior shape. After calculation, the calculated former 11 eigenvalues, the contribution rate and the cumulative contribution rate of eigenvalues are shown in Table 1, where denotes eigenvalue, denotes the contribution rate of eigenvalue and $p$ denotes the cumulative contribution rate of eigenvalue.

Table 1. Eigenvalue, Rate of Eigenvalue Contribution and Sccumulative Total Rate of Contribution

\begin{tabular}{cccccc}
\hline & 1 & 2 & 3 & 4 & 5 \\
\hline$\lambda$ & 1.0271 & 0.9978 & 0.5618 & 0.2894 & 0.1674 \\
$\tau$ & 0.2934 & 0.2850 & 0.1605 & 0.0827 & 0.0478 \\
$P$ & 0.2934 & 0.5784 & 0.7389 & 0.0826 & 0.8694 \\
\hline & 6 & 7 & 8 & 9 & 10 \\
\hline$\lambda$ & 0.1105 & 0.0878 & 0.0674 & 0.0523 & 0.0401 \\
$\tau$ & 0.0316 & 0.0251 & 0.0193 & 0.0149 & 0.0114 \\
$P$ & 0.9010 & 0.9260 & 0.9453 & 0.9603 & 0.9717 \\
\hline \multicolumn{7}{c}{111} & & & & \\
\hline$\lambda$ & 0.0307 & & & & \\
$\tau$ & 0.0088 & & & & \\
$P$ & 0.9805 & & & & \\
\hline
\end{tabular}

\subsection{Pre-segmentation}

In order to obtain better experimental contrast, two novel segmentation algorithms are proposed in this paper. The first is that we don't need the pre-segmentation before formal segmentation, and the initial contour is the circle located in the center of the image, which is named by Method One. The second is that we need the pre-segmentation on the image before formal partition, and regard the contour of pretreatment as the initial contour, which is named by Method Two. Since it does not need pre-segmentation in Method One, we introduce the pre-segmentation processing in Method Two.

The pretreatment used in this paper is that we do the expansion operation on the average SDF and make it closer to the center of the target after translation operations. The contour of the translational zero level set is shown in Fig.4. Because of the complexity of the actual image and the influence from noise and the error offset caused by automatically solving translation, we adjust the translation offset manually so that we can achieve more ideal segmentation results. 
Figure 4(a) and Figure 4(b) respectively denote the mean SDF and the contour curve of zero level set before translation. Figure 4(c) and Figure 4(d) respectively denote the mean SDF and the contour curve of zero level set after translation.

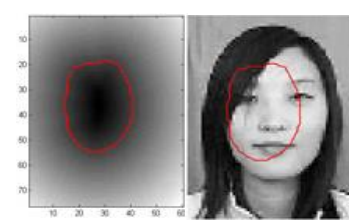

(a)

(b)

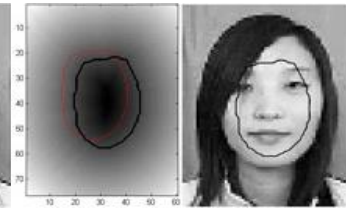

(c)

(d)

Figure 4. Effect Picture of before and after the Initial Contour of Translation

\subsection{Formal Segmentation}

We use the energy functional which described in Section 3 to guide the curve evolution whether the initial contour is circle or the preprocessed contour. Although we adjusted the pre-segmentation contour by using the method of translation in the above, we also need to do affine transformation operations in the segmentation based on the priori shape to make better contour evolution and to achieve the more ideal segmentation results.

Now we define the affine transformation parameters $T[p]$ where $p=\left[p_{1}, p_{2}, p_{3}, p_{4}\right]=\left[t_{x}, t_{y}, \theta, \rho\right], t_{x}$ and $t_{y}$ respectively denote the offset along the axis $x$ and the axis $y, \theta$ denotes the rotation angle, $\rho$ is zoom multiples. We use $\tilde{I}(\tilde{x}, \tilde{y})$ to denote the new image after affine transformation from $I(x, y)$. So we have $\tilde{I}(\tilde{x}, \tilde{y})=I(x, y)$, where

$(\tilde{x}, \tilde{y})=T[p](x, y)=\left(\rho(x \cos \theta-y \sin \theta)+t_{x}, \rho(x \cos \theta+y \sin \theta)+t_{y}\right)$

The $T[p]$ can be solved by minimizing the total energy functional $E_{\text {total }}$ via using gradient descent method. Because $E_{\text {shape }}$ has nothing to do with $p$, minimizing $E_{\text {total }}$ is equivalent to minimizing $E_{\text {image }}$. So, we obtain the formula as follows:

$\nabla_{p_{i}} E_{\text {total }}=\nabla_{p_{i}} E_{\text {image }} \quad i=1,2,3,4$

Using the gradient descent method, introducing the parameter $p$ into the image energy, we can solve the derivative of $p$ about $t$ in the image energy term which is shown as follow

$\frac{d p_{i}}{d t}=-\nabla_{p_{i}} E_{\text {image }}$

The resolution of parameter $I$ is only related to image information $T[p]$ [25].

\section{Experimental Results and Analysis}

In this section, we will use two novel segmentation algorithms proposed in this paper to do experiment, at the same time we also use the improved LBF segmentation algorithm, the extended GAC segmentation algorithm and the segmentation algorithm based on traditional PCA method to do experiments. In order to compare the advantages and disadvantages of these algorithms. 


\subsection{Experimental Environment}

The experimental environment used in the experiments described in this paper is as follows

(1) Hardware environment: Lenovo SL410k laptop, and its configuration is Intel Pentium(R) Dual-Core CPU T4500 2.3GHz; Internal storage: 2G.

(2) Software environment: The operating system is Microsoft Windows XP Professional Edition 2002 Service Pack 3; Development platform: Matlab R2010a , Visual C++ 6.0.

\subsection{Experimental Results}

Now choose the segmentation results of the following six images from test images as the experimental contrast. And Figure 5 shows the test images used for experiment, where the background of Figure 5(a) and Figure 5(b) are simple and the area of the face in Figure 5(a) is bigger than that in Figure 5(b), the position of the face is a little to the right in Figure 5(c), the part of the face is shielded by the external things in Figure 5(d), the backgroud of Figure 5(e) and Figure 5(f) are complicated.

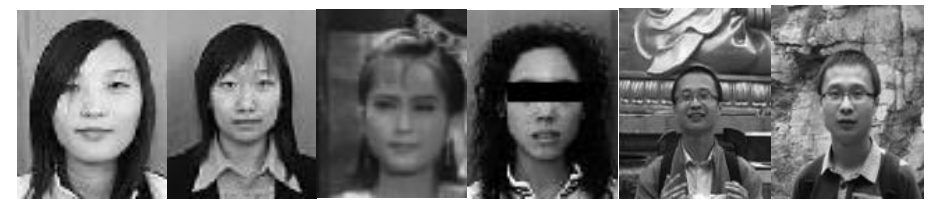

(a) (b)

(c)

(d)

(e)

(f)

Figure 5. Test Image

Figure 6 shows the initial contour of the Method One proposed in this paper and the extended GAC segmentation model, the improved LBF segmentation model, the segmentation model based on traditional PCA method. The initial contour of these experiments is circle, which the initial position of is basically located in the center of the image.

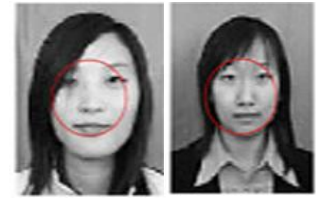

(a) (b)

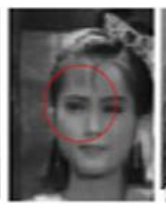

(c)

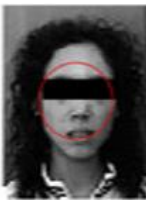

(d)

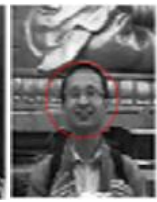

(e)

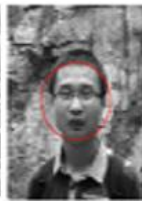

(f)

Figure 6. Initial Contour

Fig.7 shows the segmentation results of the extended GAC, where the parameters are set to: $\sigma=2$ (Gaussian Kernel Parameter), $c=3$ (The chosen constant velocity coefficient), $k s=3$ (Gaussian Kernel Size, for Odd Number), and the iterations $\mathrm{Nb}$ _iter $=150$. 


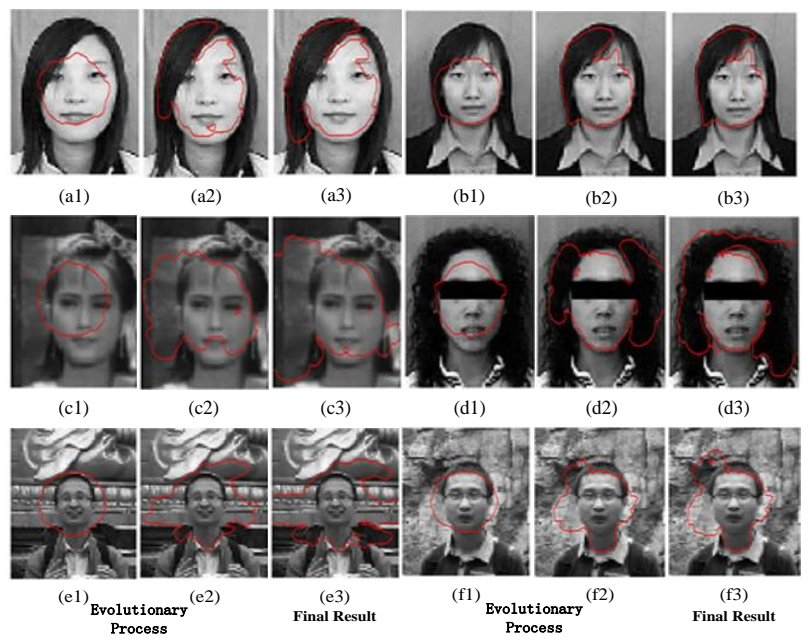

Figure 7. Segmented Results of Extended GAC

Figure 8 shows the segmentation results of the improved LBF, where the parameters are: $\quad \lambda_{1}=1.0, \lambda_{2}=1.0 \quad$ (or $\quad \lambda_{1}=1 \quad, \quad \lambda_{2}=2 \quad$ ), $\quad v=0.001 * 255 * 255$ (or $v=0.002 * 255 * 255, v=0.003 * 255 * 255, v=0.004 * 255 * 255$ ), $\beta=1, \sigma=3$ ( Gaussian Kernel Parameter), $\varepsilon=1$ ( Parameter of Heaviside function and Dirac function), and the iterations $\mathrm{Nb}$ _iter $=150$.

In Figure 7 and Figure 8, the first, second, fourth, and fifth column denote parts of the evolution process of the two segmentation models, and the third, sixth column denote the final segmentation results of the two kinds of segmentation models. It can be seen from Figure 7 and Figure 8 that because the extended GAC model and the improved LBF model have no prior shape, the segmentation results are not ideal for the complex images.

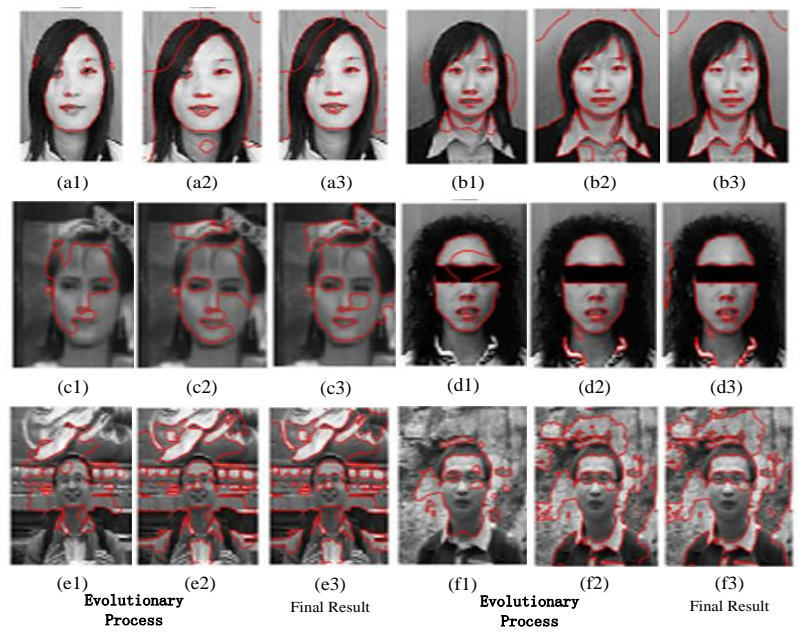

Figure 8. Segmented Results of Improved LBF

In order to solve the problem of the under segmentation or the over-segmentation problem shown above, some scholar introduced the prior shape, and used PCA method to decrease dimensions of the training samples to eliminate the redundant information of the training samples, which is named by MS_GAC_SHAPE. Relevant parameters can be set: $\alpha_{s}=5$ (Coefficient in front of the Shape Item), $\alpha_{b}=1$ (Coefficient in front of the Boundary Item), $\alpha_{r}=100$ (Coefficient in front of the Regional Item), $\mu=25, \theta=0$. 
The degree of fitting which is used in PCA method to deal with the training samples is 0.98. The segmentation results are shown in Figure 9.

In Figure 9, the first, second, fourth, and fifth column denote parts of the evolution results, and the third, sixth column denote the final segmentation results. It can be seen from Figure 9 that the traditional PCA method overcome the segmentation problem occurred in the extended GAC and the improved LBF to a certain extent. The PCA method belongs to the linear principal component analysis, and builds the new sample sets with linear expression in the process of dimension reduction. However, all of the training sample sets of the images are not necessary linear in the application. In some cases, the segmentation results are not ideal, such as in Figure 9 (e3)and Figure 9(f3).

We proved the limitations of the extended GAC, improved LBF and traditional PCA segmentation method in Figure 7, Figure 8 and Figure 9. We use the method described in Section 3 to conduct an experiment. $\Delta t=0.1, \varepsilon=1$. The fitting used by KPCA to deal with the training sample sets is 0.98 . There are two groups of experiments in this section. The difference between the two groups of experiments is that the initial contour is fixed circular (named by LBF_KPCA_Circle) in the first group, whose position is located in the center of image, and the initial contour is the contour after pre-segmentation(named by LBF_KPCA_Init) in the second group. The method of pre-segmentation has been described detailedly in Section IV.

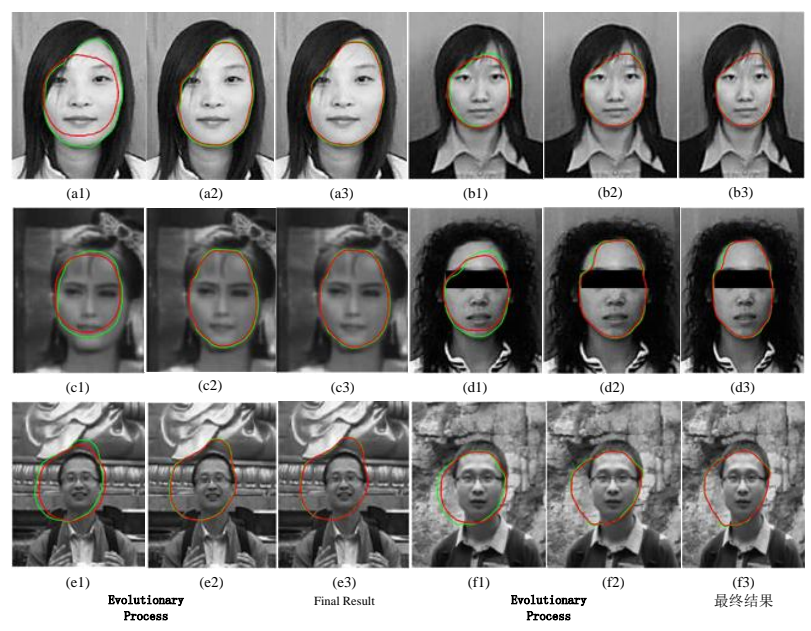

Figure 9. Results of Traditional PCA Segmentation

We will show the experiments of the first group (LBF_KPCA_Circle). Because the features and pixel values are different of each image in the process of the real experiment, the specific parameters setting are different in the actual process of the segmentation. The parameters settings of the test images in Figure 5 are shown in Table 2, where $\sigma$ is the parameter of Gauss Radial Kernel Function, $\alpha$ is the weighting coefficient which weighs the image energy and the shape energy. 
Table 2. LBF_KPCA_Circle Parameter Setting

\begin{tabular}{lcccccc}
\hline & $\lambda_{1}$ & $\lambda_{2}$ & $v$ & $\beta$ & $\sigma$ & $\alpha$ \\
\hline Figure 5(a) & 1.0 & 1.0 & $0.004 * 255 * 255$ & 1.0 & 245 & 0.55 \\
Figure 5(b) & 1.0 & 1.0 & $0.001 * 255 * 255$ & 1.0 & 245 & 0.35 \\
Figure 5(c) & 1.0 & 2.0 & $0.001 * 255 * 255$ & 1.0 & 245 & 0.775 \\
Figure 5(d) & 1.0 & 1.0 & $0.002 * 255 * 255$ & 1.0 & 245 & 0.725 \\
Figure 5(e) & 1.0 & 4.0 & $0.215 * 255 * 255$ & 1.0 & 245 & 0.79 \\
Figure 5(f) & 1.0 & 3.0 & $0.215 * 255 * 255$ & 1.0 & 245 & 0.818 \\
\hline
\end{tabular}

According to parameter settings in Table 2, we made experiments on the test images in Figure 5, and the segmentation results is shown in Figure 10.

In Figure 10, the first, second, fourth, and fifth column denote parts of the evolution results, and the third, sixth column denote the final segmentation results. It can be seen from Figure 9 that the new method proposed in this paper has better segmentation effect on the images with complex background and noise, the segmentation effect is better than that used the extended GAC segmentation model, the improved LBF segmentation model, and the segmentation model based on the traditional PCA.
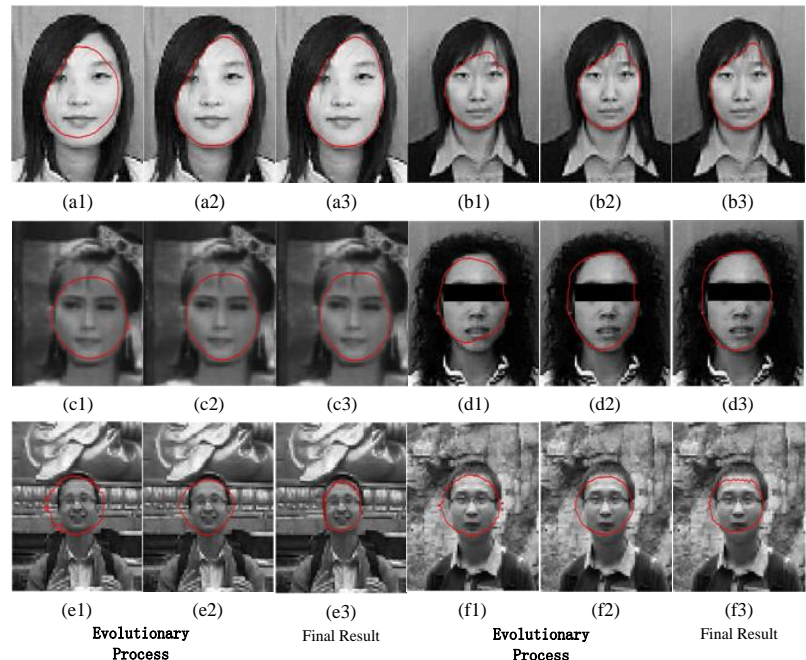

Figure 10. Segmentation Results of LBF_KPCA_Circle

The image energy chosen is the improved LBF model, the initial position has certain influence on the segmentation result of the image. So the second method (namely LBF_ KPCA _Init) will continue to be described in the following, which means that does presegmentation processing before formal partition of which the specific methodology is firstly do the expansion operation on the average SDF, then make it closer to the center of the target after translation operations. Finally, we use the contour after the presegmentation as the initial contour of formally segment. Because the gray level information and characteristics of the image is different, the parameter settings in our experiment can't be exactly same. After attempting to contrast, the parameter settings of the test images in Figure 5 are shown in Tab.3. Tx, Ty are respectively the offset on the $x$ direction and the $y$ direction (Providing that the origin of coordinates of the image is located on the top left corner of the computer screen, the right direction is the positive 
direction on $x$ direction, and the downward direction is the positive direction on $y$ direction).

According to parameter settings in Table 3, we experimentalize the test images in Figure 5, and the segmentation results is shown in Figure 11.

The first and the fourth column in Fig.11 are the initial contour, namely the SDFs which are obtained by the dilation operation, the second and the fifth column are the adjusted contour, namely the SDFs which are obtained by the translation operation on the SDFs obtained by the expanding operation, and the third and the sixth column are the final segmentation results.

Table 3. LBF_KPCA_Init Parameter Setting

\begin{tabular}{lcccccccc}
\hline Parameters & $\lambda_{1}$ & $\lambda_{2}$ & $v$ & $\beta$ & $\sigma$ & $\alpha$ & $T x$ & $T y$ \\
\hline Figure 5(a) & 1.0 & 1.0 & $0.004 * 255 * 255$ & 1.0 & 245 & 0.55 & 5 & 3 \\
Figure 5(b) & 1.0 & 1.0 & $0.001 * 255 * 255$ & 1.0 & 245 & 0.35 & 5 & 2 \\
Figure 5(c) & 1.0 & 2.0 & $0.006 * 255 * 255$ & 1.0 & 245 & 0.65 & 6 & 5 \\
Figure 5(d) & 1.0 & 2.0 & $0.002 * 255 * 255$ & 1.0 & 245 & 0.85 & 4 & 4 \\
Figure 5(e) & 1.0 & 4.0 & $0.215 * 255 * 255$ & 1.0 & 245 & 0.70 & 3 & 1 \\
Figure 5(f) & 1.0 & 4.0 & $0.215 * 255 * 255$ & 1.0 & 245 & 0.70 & 3 & 1 \\
\hline
\end{tabular}

It can be seen from Fig.11 that the new method proposed has a better segmentation result on the images with complex background image and big noise, the segmentation results are better than that used by the extended GAC model, the improved LBF model, the segmentation model based on PCA and the LBF_KPCA_Circle proposed in this paper.
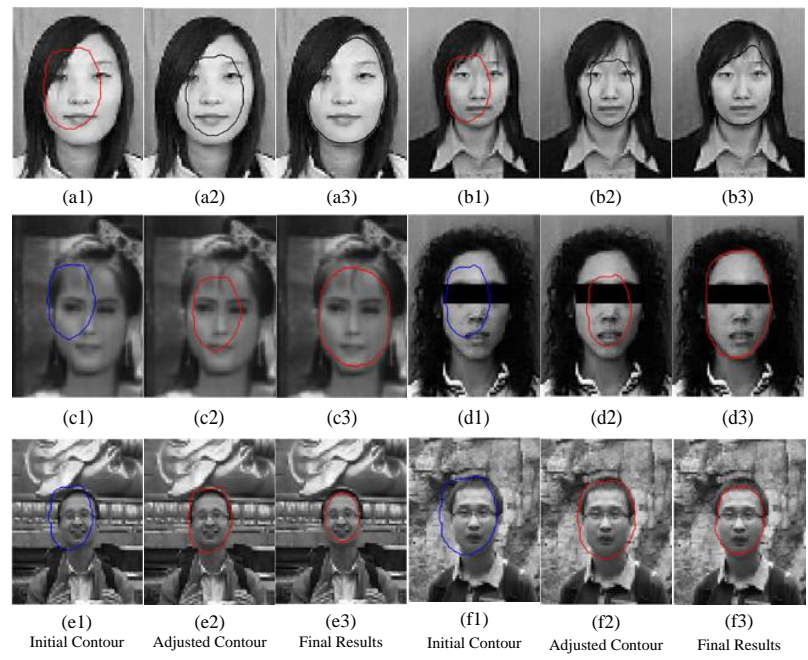

Figure 11. Segmentation Results of LBF_KPCA_Init

\subsection{Evaluation and Analysis of Segmentation Results}

We mainly analyze and evaluate the experimental results in Section B from the segmentation in time and accuracy.

In this section, we analyze the problems of time efficiency, the detailed description is as follows.

We compare the segmentation efficiency of the segmentation method based on the traditional PCA (MS_GAC_SHAPE) and two kinds of novel proposed segmentation methods, namely LBF_ KPCA_Circle segmentation method and LBF_KPCA _Init segmentation method. 
Through experiments and analysis, the statistical graph of the time efficiency of three kinds of segmentation method is shown in Figure 12, where the black line with circles denotes the time statistics used in the segmentation model based on the traditional PCA, the red line with $(+)$ denotes the time statistics used in the first segmentation method (LBF_KPCA_Circle) proposed in this paper, and the blue line with $(*)$ denotes the time statistics used in the second method (LBF_KPCA_Init) proposed in this paper.

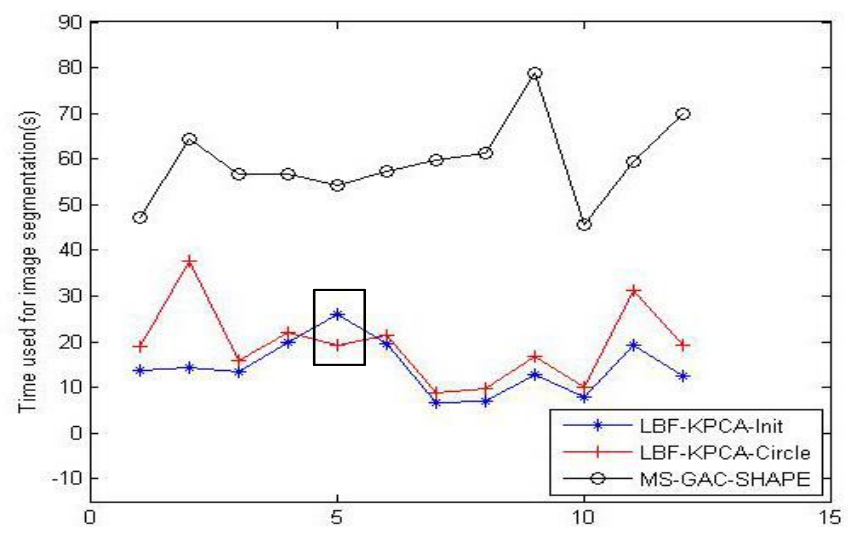

Figure 12. Time Statistical Results of Three Algorithms

It can be seen from Figure 12 that the segmentation time of the two novel proposed segmentation methods is much faster than that of the segmentation method based on the traditional PCA, the reasons are as follows:

(1) The reasons why the segmentation time of the segmentation method based on the traditional PCA is too long are as follows: In order to guarantee the properties of SDF in the curve evolution process, it is needed to re-initialize the SDF every once in a while and the process will take a long time. Also, the energy term adopted in the segmentation model based on the traditional PCA is based on global information, so it takes some time to judge and dispose in the process of the curve evolution.

(2) The reasons why the time used by the two novel segmentation methods is shorter than that used by the segmentation model based on the traditional PCA are as follows: There is no need to re-initialize the SDF in the process of curve evolution in the two novel methods, in other words, these methods add the penalty term into the image energy to assure the evaluative curve always keeping as the SDF in the process of evolution which saves the time used by continuously re-initializing the SDF in the segmentation model based on the traditional PCA. Moreover the image energy term adopted by the two kinds of novel segmentation methods is based on the improved LBF model which is based on local information, so these methods are faster than that based on global information in the time efficiency.

(3) The segmentation efficiency of LBF_KPCA_Init method is faster than that of LBF_KPCA_Circle method. In Figure 12 the red line with (+) and the blue line with $(*)$, the important reason is the pre-segmentation on the target before the formal segmentation in LBF_KPCA_Init method which is more helpful to segment the image by adopting the proposed energy functional. But not all segmentation efficiency of LBF_KPCA_Init method on every image is superior to that of LBF_KPCA_Circle method, such as the rectangular box in Figure 12. The reason may be that the initial contour of LBF_KPCA_Circle method is much closer to the target than that of LBF_KPCA_Init method.

In the following, we will use the Similarity Index (SI)[25], the Relative Difference Degree (RDD) and the Relative Overlap Degree (ROD)[26] to compare the extended 
GAC segmentation method, the improved LBF segmentation method, MS_GAC_SHAPE segmentation method, LBF_KPCA_Circle segmentation method and comparison LBF_KPCA_Init segmentation method on the segmentation accuracy.

The formulas of the Similarity Index (SI) is defined by (34)

$S I=\frac{2 N\left(C_{1} \cap C_{2}\right)}{N\left(C_{1}\right)+N\left(C_{1}\right)}$

where $N(C)$ denotes the number of pixels contained by the contour curve $C$ in the binary image, $C_{1}$ and $C_{2}$ respectively denote the contour curve $O S$ of the segmentation result in our experiments and the contour curve of the referred segmentation result, namely the so-called "gold standard" $O T, N\left(C_{1} \cap C_{2}\right)$ denotes the number of pixels of the intersecting part between $C_{1}$ and $C_{2}$. The evaluation standard provides that the greater the value of SI, the segmentation results closer to the "gold standard" $O T$.

The Relative Difference Degree (RDD) and the Relative Overlap Degree (ROD) is defined by (35).

$$
\begin{aligned}
& R D D=\frac{\left|O_{T}\right|-\left|O_{S}\right|}{\left|O_{T}\right|} \times 100 \% \\
& R O D=\min \left\{\frac{\left|O_{T} \cap O_{S}\right|}{\left|O_{T}\right|}, \frac{\left|O_{T} \cap O_{S}\right|}{\left|O_{S}\right|}\right\}
\end{aligned}
$$

where $\left|O_{T}\right|$ and $\left|O_{S}\right|$ respectively denote the number pixels in the inner of the referential contour curve and the number pixels in the inner of the experimental contour curve, $\left|O_{T} \cap O_{S}\right|$ denotes the number of pixels of the intersecting part between the inner of the referential contour curve and that of the experimental contour curve. If the $O S$ is exactly the same to the $O T$, the value of ROD is $100 \%$ and the value of RDD is 0 , or the value of ROD is less than $100 \%$. So the greater value of ROD is, the closer the segmentation result is to the $O T$ in the practical application.

\begin{tabular}{|c|c|c|c|c|c|c|c|c|c|}
\hline & \multicolumn{3}{|c|}{ Figure 5(a) } & \multicolumn{3}{|c|}{ Figure 5(b) } & \multicolumn{3}{|c|}{ Figure 5(c) } \\
\hline & $S I$ & $R O D$ & $R D D$ & $S I$ & $R O D$ & $R D D$ & $S I$ & $R O D$ & $R D D$ \\
\hline Extended GAC & 0.2378 & 0.3526 & $\begin{array}{c}- \\
0.4329\end{array}$ & 0.2873 & 0.3787 & $\begin{array}{c}- \\
0.4023\end{array}$ & 0.1527 & 0.3787 & $\begin{array}{c}- \\
0.4903\end{array}$ \\
\hline Improved $L B F$ & 0.3789 & 0.4529 & $\begin{array}{c}- \\
0.2426\end{array}$ & 0.2973 & 0.3673 & -0.235 & 0.1627 & 0.3673 & $\begin{array}{c}- \\
0.5123\end{array}$ \\
\hline$M S \_G A C \_S H A P E$ & 0.9683 & 0.9616 & 0.0290 & 0.9504 & 0.9515 & 0.0310 & 0.9742 & 0.9702 & 0.0234 \\
\hline LBF_KPCA_Circle & 0.9759 & 0.9724 & 0.0218 & 0.9788 & 0.9757 & 0.0231 & 0.9756 & 0.9789 & 0.0201 \\
\hline \multirow[t]{3}{*}{$L B \bar{F} \_K P C \bar{A} \_$Init } & 0.9867 & 0.9849 & 0.0189 & 0.9857 & 0.9843 & 0.0182 & 0.9803 & 0.9802 & 0.0103 \\
\hline & \multicolumn{3}{|c|}{ Figure $5(d)$} & \multicolumn{3}{|c|}{ Figure 5(e) } & \multicolumn{3}{|c|}{ Figure 5(f) } \\
\hline & $S I$ & $R O D$ & $R D D$ & $S I$ & $R O D$ & $R D D$ & $S I$ & $R O D$ & $R D D$ \\
\hline Extended GAC & 0.1723 & 0.2355 & 0.5126 & 0.4013 & 0.4579 & $\begin{array}{c}- \\
0.1315\end{array}$ & 0.2803 & 0.2707 & $\begin{array}{c}- \\
0.4510\end{array}$ \\
\hline Improved $L B F$ & 0.1833 & 0.2474 & $\begin{array}{c}- \\
0.5104\end{array}$ & 0.3014 & 0.3578 & 0.2389 & 0.1604 & 0.2079 & $\begin{array}{c}- \\
0.5210\end{array}$ \\
\hline MS_GAC_SHAPE & 0.9454 & 0.9347 & 0.0325 & 0.8854 & 0.8765 & $\overline{-}-\overline{0434}$ & 0.7848 & 0.7985 & 0.0500 \\
\hline LBF_KPCA_Circle & 0.9625 & 0.9458 & 0.0213 & 0.9354 & 0.9427 & 0.0379 & 0.9321 & 0.9477 & 0.0303 \\
\hline$L B \bar{F} \_K P C A \_I n i t$ & 0.9824 & 0.9759 & 0.0131 & 0.9753 & 0.9678 & 0.0287 & 0.9877 & 0.9788 & 0.0110 \\
\hline
\end{tabular}

Table 4. Segmentation Evaluation Results 


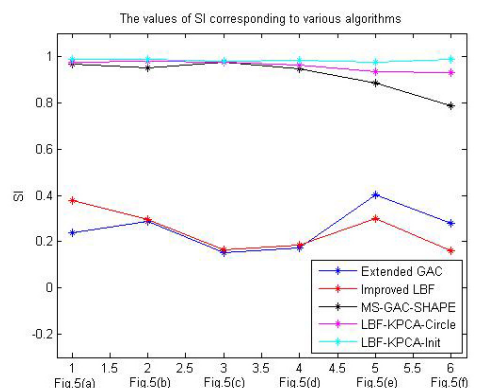

(a)

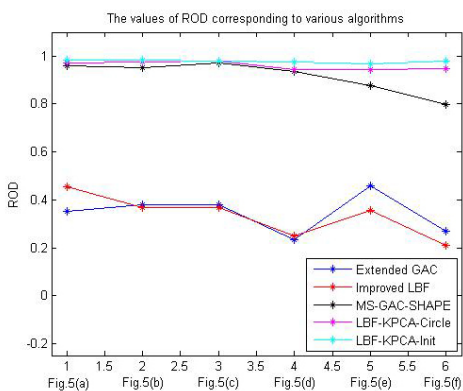

(b)

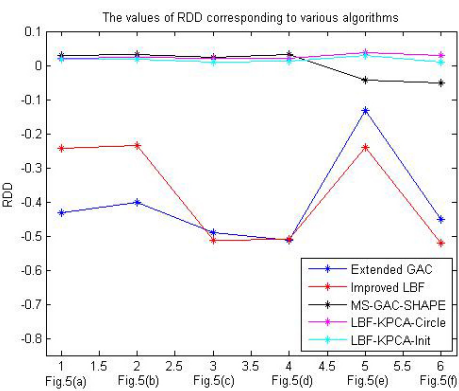

(c)

Figure 13. The Statistical Diagram of the Segmentation Evaluation Results

The values of various evaluation parameters corresponding to different segmentation methods are shown in Table 4, the computed results are kept four decimal places, and the statistical diagram of the values of all evaluation parameters are shown in Figure 13.

Figure 13(a), Figure 13(b), and Figure 13(c) respectively show the statistical situation of the evaluation standard SI, ROD and RDD, where the ordinate denotes the value size corresponding the parameters, the integers from 1 to 6 on the abscissa respectively denote Figure 5(a), Figure 5(b), Figure 5(c), Figure 5(d), Figure 5(e) and Figure 5(f)

It can be seen from Table 4 that the segmentation accuracy of the segmentation model based on the traditional PCA (MS_GAC_SHAPE segmentation method) is superior to the extended GAC segmentation method and the improved LBF segmentation method. Because the method based on the traditional PCA introduces the priori shape to guide the evolution of the curve, which can make the method have a good segmentation effect on most images.

The segmentation model based on the traditional PCA uses the linear expression to deal with the training samples. The vast majority of shapes are nonlinear in the actual image, so the segmentation method based on the traditional PCA has bad segmentation results while segmenting parts of images, for example Figure 9(e) and Figure 9(f). Futhermore, the initial contour of the segmentation method based on the traditional PCA is fixed circular which makes the pixel values similar to the target and affects the normal segmentation of the curve in the evolution process.

It can be seen from the experimental comparison and the parameter values in Table 4 that the segmentation accuracy of the two kinds of novel methods is superior to that of the segmentation method based on the traditional PCA, the reasons are as follows.

(1) The kernel principal component analysis (KPCA) is adopted to reduce dimensions of the training samples which makes the eigenvalues and eigenvectors of the training set express the prior shape preferably and makes the prior shape guide the curve evolution preferably.

(2) We do the pre-segmentation on the target before the formal segmentation in the second novel method (LBF_KPDA_Init segmentation method) which avoids the pixel values similar to the target and affects the curve evolution.

\section{Conclusion}

The Kernel Principal Component Analysis method is adopted to decrease dimensions of the training samples, solve the linearized limitations caused by the segmentation method based on the traditional PCA and propose the shape energy based on the feature space which is combined the improved LBF model. The proposed method efficiently utilizes shape information of face images, therefore it is able to segment facial images under complex background or partially occluded. Experimental results also demonstrate desirable performance of our method. Via the comparison of several groups of experiments, it is proved that the segmentation precision and efficiency of the novel level set segmentation method based on prior shape are improved. 


\section{Acknowledgements}

This work is supported by Education Department of Liaoning Province under Grant No.2010076. The authors also gratefully acknowledge the helpful comments and suggestions of the reviewers, which have improved the presentation.

\section{References}

[1] C. Li, C. Xu, C. Gui.et al.. "Level set evolution without re-initialization: A new variational formulation". in IEEE Conference on Computer Vision and Pattern Recognition (CVPR), (2005), pp. 430-436.

[2] N. Paragios, R. Deriche. "Geodesic active regions and level set methods for supervised texture segmentation". International Journal of Computer Vision, (2002), vol.46, no.3, pp. 223-247.

[3] V. Caselles, R. Kimmel, and G. Sapiro. "Geodesic active contours". International Journal of Computer Vision, (1997), vol.22, no.1, pp.61-79.

[4] Mumford D and Shah J. "Optimal approximations by piecewise smooth functions and associated variational problems". Communications on Pure and Applied Mathematics, (1989), Vol.42, No.5, pp.577-685.

[5] L. Vese, T. Chan. "A Multiphase Level Set Framework for Image Segmentation Using the Mumford and Shah Model”. International Journal of Computer Vision, (2002), vol.50, no.3, pp.271-293.

[6] T. Chan, L. Vese. "Active contours without edges". IEEE Transactions on Image Processing, (2001), vol.10, no.2, pp.266-277.

[7] Li C, Kao C Y, Gore J C, et al. "Implicit Active Contours Driven by Local Binary Fitting Energy". in IEEE Conference on Computer Vision and Pattern Recognition, Minneapolis, MN, (2007), pp.1-7..

[8] P. H. Lim, U. Bagci, O. Aras, et al. "A novel spinal vertebrae segmentation framework combining geometric flow and shape prior with level set method". in IEEE International Symposium on Biomedical Imaging, Barcelona, (2012), pp.1703-1706.

[9] S. Chen, R. J. Radke. "Level Set Segmentation with Both Shape and Intensity Priors". in IEEE International Conference on Computer Vision, Kyoto, (2009), pp.763-770.

[10] V. A. Prisacriu, I. Reid. "Nonlinear Shape Manifolds as Shape Priors in Level Set Segmentation and Tracking”. In IEEE Conference on Computer Vision and Pattern Recognition, Providence, RI, (2011), pp.2185-2192.

[11] O. Gloger, V. Liebscher, B. Kugelmann, et al. "Prior Shape Level Set Segmentation on Multistep Generated Probability Maps of MR Datasets for Fully Automatic Kidney Parenchyma Volumetry". IEEE Transactions on Medical Imagin , (2012), vol. 31no.2, pp.312-325.

[12] F. Khalifa, A. El-Baz, R. Ouseph, et al. "Shape-Appearance Guided Level-Set Deformable Model for Image Segmentation". in IEEE International Conference on Pattern Recognition, Istanbul, (2010), pp.4581-4584.

[13] S. Y. Yeo, X. Xie, I. Sazonov, et al. "Level Set Segmentation with Robust Image Gradient Energy and Statistical Shape Prior". In IEEE International Conference on Image Processing, Brussels, (2011), pp.3397-3400.

[14] X. Q. Wu, K. Shishir. Shah. "Cell segmentation in multispectral images using level sets with priors for accurate shape recovery". In IEEE International Symposium on Biomedical Imaging, Chicago, IL, (2011), pp.2117-2120.

[15] J. Y. Dong, C. Y Hao. "Review of Statistical Shape Prior-based Level Set Image Segmentation". Computer Science, (2010), vol.37, no.1, pp.6-9.

[16] J. X. ZHENG, L. YANG, Z. K. WANG. "Combining prior shape and Mumford-Shah model for active contour segmentation". Computer Engineering and Applications, (2011), vol.47, no.23, pp.167-170.

[17] L. P. YANG, Q. ZOU. "Level Set Image Segmentation Method Based on Prior Shape Knowledge". Computer Science, (2012), vol.39, no.8, pp. 288-291.

[18] J. Mercer. "Functions of Positive and Negative Type and Their Connection with the Theory of Integral Equations". Proceedings of the Royal Society A: Mathematical, Physical and Engineering Sciences, (1909), vol.83, no.559, pp. 69-70.

[19] J. Kwok, I. Tsang. "The Pre-Image Problem in Kernel Methods". IEEE Transactions on Neural Networks, (2004), vol.15, no.6, pp.1517 - 1525.

[20] S. Mika, B. Scholkopf, A. J. Smola, et al. "Kernel PCA and De-Noising in Feature Space". In Proceedings of the 1998 conference on Advances in neural information processing systems II, MIT Press Cambridge, MA, USA, (1999), pp.536-542.

[21] S. Dambreville, Y. Rathi, A. Tannenbaum, et al. "A Framework for Image Segmentation Using Shape Models and Kernel Space Shape Priors". IEEE Transactions on Pattern Analysis and Machine Intelligence, (2008), vol.30, no.8, pp.1385 - 1399.

[22] N. Paragios, M. Rousson, V. Ramesh. "Matching Distance Functions: A shape-to-area variational approach for global-to-local registration". In ECCV '02 Proceedings of the 7th European Conference on Computer Vision, Springer-Verlag London, (2002), pp.775-789. 
[23] M. Leventon, E. Grimson, O. Faugeras. "Statistical shape influence in geodesic active contours". In IEEE International Conference on Computer Vision and Pattern Recognition, Hilton Head Island, SC, (2000), pp.316-323.

[24] A. Tsai, A. J. Yezzi, C. Tempany, et al. "A shape-based approach to the segmentation of medical imagery using level sets". IEEE Transactions on Medical Imaging, (2003), vol.22, no.2, pp.137 - 154,.

[25] A. P. Zijdenbos, B. M. Dawant, R. A. Margolin. "Morphometric Analysis of White Matter Lesions in MRI Images: Method and Validation". IEEE Transactions on Medical Imaging, (1994),vol.13, no.4, pp.716- 724

[26] D. L. Collins, A. C. Evans, C, Holms. "Automatic 3D segmentation of neuro-anatomical structures from MRI". In Information Processing in Medical Imaging In 14th International Conference on Information Processing in Medical Imaging, LNCS, (1995), pp.139-152.

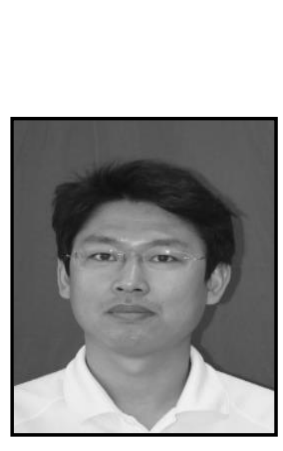

Authors

Ji Zhao is a professor in School of Software Engineering, University of science and technology Liaoning. He received the master degree in computer application technology in 2004 and $\mathrm{Ph} . \mathrm{D}$. degree in detection technology and automation equipment in 2013, both from Northeastern University China. His research interests include image segmentation and embedded technology and application. His present interests are expanded to face's localization, segmentation and expression understanding. He was a Visiting Researcher at Saitama University of industry, Japan in 2012. He was awarded the Liaoning Province Natural Science Academic Achievement prize from Liaoning science and Technology Association of China and Anshan Natural Science Academic Achievement prize from Anshan science and Technology Association of China in 2012. 
International Journal of Signal Processing, Image Processing and Pattern Recognition Vol. 10, No. 2 (2017) 